\title{
Incidences de la notion de cours d'eau sur la gestion et la pérennité des étangs. Le cas de la dérivation
}

Impacts of watercourse concept on the management and sustainability of ponds: the case of the derivation

Die Auswirkungen des Konzept der Fließgewässer auf das Management und die Nachhaltigkeit von Teichen : der Fall der Umleitung

\section{Camille Millot}

\section{(2) OpenEdition}

\section{Journals}

Electronic version

URL: http://journals.openedition.org/rge/5803

ISSN: 2108-6478

Publisher

Association des géographes de l'Est

\section{Printed version}

Date of publication: 22 April 2016

ISSN: 0035-3213

Electronic reference

Camille Millot, «Incidences de la notion de cours d'eau sur la gestion et la pérennité des étangs. Le cas de la dérivation », Revue Géographique de l'Est [Online], vol.56 / n¹-2 | 2016, Online since 29 August 2016, connection on 08 September 2020. URL : http://journals.openedition.org/rge/5803

This text was automatically generated on 8 September 2020.

Tous droits réservés 


\title{
Incidences de la notion de cours d'eau sur la gestion et la pérennité des étangs. Le cas de la dérivation
}

\author{
Impacts of watercourse concept on the management and sustainability of ponds: \\ the case of the derivation \\ Die Auswirkungen des Konzept der Fließgewässer auf das Management und die \\ Nachhaltigkeit von Teichen : der Fall der Umleitung
}

\section{Camille Millot}

1 «Pourquoi la présence des étangs pose tant de problèmes en France ?! ». Cette question, nous l'avons entendue de la bouche de chercheurs estoniens - limnologues et biologistes - après chaque exposé de la problématique des étangs en France $^{1}$. Ces scientifiques n'arrivaient pas à concevoir l'existence ou l'origine des débats houleux que peut susciter cet objet, quand bien même les différents arguments juridiques, environnementaux et les résultats scientifiques leurs aient été exposés. Cette interrogation soulevée par les estoniens n'existe pas en France. Les discours sont là : « les étangs sont trop nombreux $»^{2} ;$ «ils dégradent la qualité de l'eau $»^{3} ;$ «ils rompent la continuité écologique du cours d'eau et arrêtent le transport naturel des sédiments de l'amont vers l'aval d'un bassin versant et la circulation des espèces piscicoles " (ONEMA, 2013). Pour autant, les fondements scientifiques de ces discours en faveur de la destruction des étangs sont maigres. Le recul de ces chercheurs étrangers sur cette situation nous indique une chose essentielle: quel que puisse être l'impact environnemental d'un étang, le "problème » des étangs n'est pas une réalité en soit, encore moins un axiome, il est une construction sociopolitique que la recherche se doit d'interroger. La nature des enjeux relevant aussi bien de questions physiques (qualité de l'eau) que de questions sociales (protection du patrimoine commun, choix réglementaires), la géographie trouve toute sa place en étudiant les étangs de manière systémique. 
2 Au vu des discours à l'origine des objectifs de gestion contemporaine des étangs ainsi que des discours en faveur de leur disparition (ONEMA, 2010 a), cette construction est liée avant toute chose à la perception des «masses d'eau ", patrimoine fragile devant être protégé. Effectivement, la réglementation appliquée aux étangs est fortement dépendante des cours d'eau. Ceci s'applique par exemple pour la détermination en eaux closes ou libres, des quantités de MES rejetées en aval, du débit réservé et des catégories de poissons autorisées.

3 Les étangs sur cours d'eau, par leurs configurations et leurs usages, modifient la qualité physico-chimique et la continuité écologique des cours d'eau, du moins temporairement. Or, ces critères sont la clef de voute de la réglementation française actuelle sur les cours d'eau. "Alternative à leur suppression », une "solution » à leur maintien semble être à la portée des aménageurs et propriétaires d'étangs: la dérivation. Notons une fois de plus le vocable usité et le terme de "solution », associé de manière systématique à la dérivation. Ceci démontre bien que l'étang n'est pas envisagé comme une création anthropique aménageable mais comme un problème à corriger. Le principe de cet aménagement est de détourner une partie de cours d'eau en amont de l'étang par un canal qui contourne cet étang et rejoint son exutoire. Il existe deux modes possibles (Fig. 1).

Figure 1 : Le principe des étangs à dérivation ${ }^{5}$

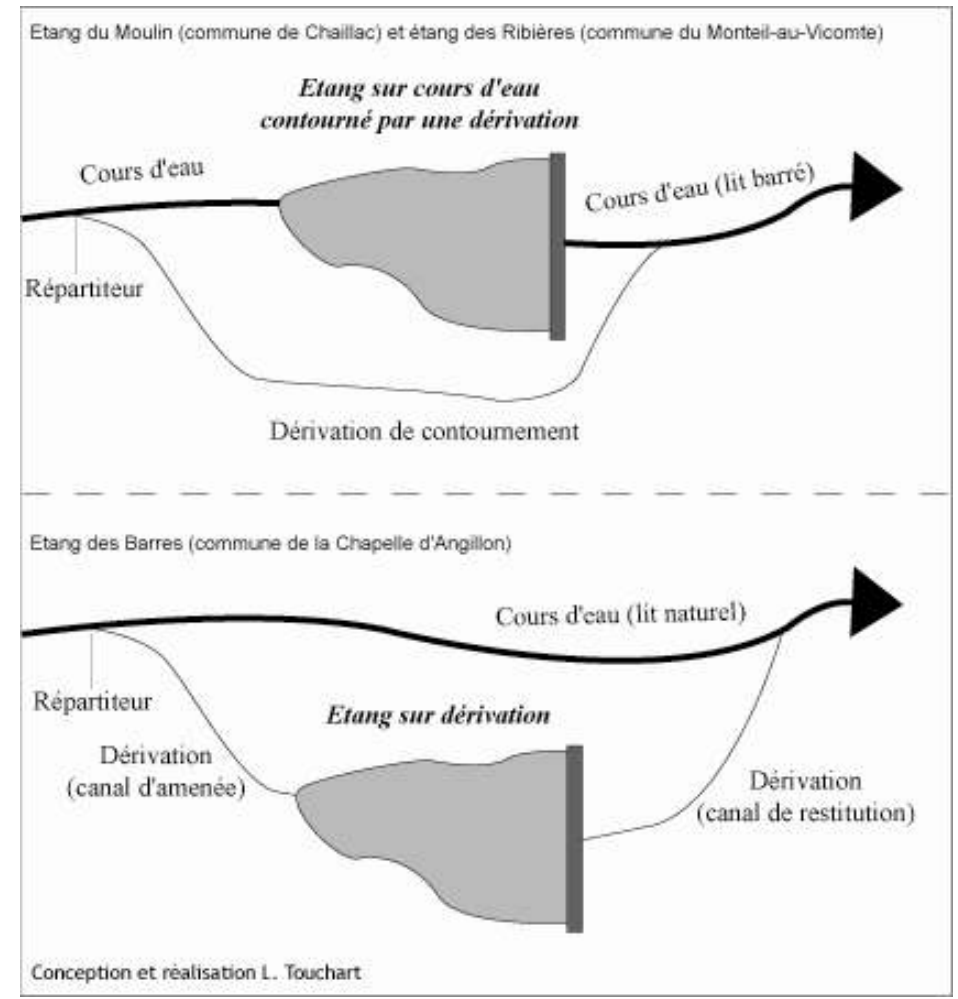

4 L'objectif est de limiter les impacts de l'étang, cités plus avant, sur l'eau du cours d'eau aval. La théorie est intéressante mais il n'en reste pas moins que les impacts socioéconomiques et environnementaux de la dérivation sont méconnus, pour ne pas dire inconnus. 
Quelles sont les conséquences de la réglementation et des aménagements induits pour l'avenir des étangs? Une gestion optimale est-elle réalisable ou sont-ils amenés à disparaître?

Les étangs ne sont pas un objet de laboratoire à part entière mais sont partie intégrante des hydrosystèmes. Pour étudier la gestion et l'aménagement des étangs, il faut en premier lieu réfléchir à une question essentielle: que considère-t-on comme cours d'eau en France ? L'aspect réglementaire de la question sera ensuite discuté, par la mise en perspective que permet l'étude de terrain. Enfin, il faut étudier la dérivation sous ses différents aspects pour comprendre les conditions où elle peut représenter un aménagement optimal favorisant la pérennité des étangs.

\section{I - Les cours d'eau : noyau de la réglementation sur l'eau}

7 Nombreux sont les termes géographiques qui sont aujourd'hui ce qu'on appelle communément des mots valises. Les termes perdent leur signification initiale et spécifique, soit parce que les objets auxquels ils sont attachés sont mal identifiés, soit parce qu'une méconnaissance de la signification du terme incite à une utilisation abusive de celui-ci. Il en va ainsi du territoire, du climat ou de la météorologie entre autres. De même, communément, le "cours d'eau" ne désigne pas un objet spécifique mais un fossé dans lequel de l'eau s'écoule : rivière, source, fleuve. La Directive Cadre Européenne sur l'eau n'en fait pas mention. Elle prend le terme plus général encore de masse d'eau de surface. La généralité du terme ou la possibilité ou non de déterminer un cours d'eau n'auraient aucune incidence particulière si une réglementation - elle, très spécifique - n'y était pas attachée, déterminant la gestion des hydrosystèmes et de leurs aménagements.

\section{A - Droit de l'eau français, directives européennes et conceptions du cours d'eau : de la définition de l'usage à sa protection environnementale}

8 Le premier texte moderne concernant le droit de l'eau remonte à l'ordonnance sur les eaux et forêts de Colbert en 1669. Par la suite, les codes napoléoniens précisent le droit de l'eau dont " l'objectif principal était de déterminer le régime de propriété de l'eau » (eaufrance.fr). La domanialité se déterminait alors principalement par rapport au caractère navigable du cours d'eau. Il y avait donc d'un côté des biens affectés et de l'autre des choses communes. C'est ainsi que pour un même objet, le code civil a fait « coexister un droit tourné vers l'exploitation économique, et un autre orienté vers le partage » (Marc, 2006). Toutefois, comme le note l'auteur, «la notion de patrimoine commun de la nation introduite par le droit de l'environnement invite à relativiser cette dualité statutaire. Le droit de l'environnement, dont l'objectif est de préserver les ressources naturelles fait l'économie de ces distinctions civiles » (loc. cit.). De nouveaux critères de classement sont introduits avec en premier lieu la qualité des eaux, qui "s'est affirmée comme un nouveau critère d'ordonnancement juridique des cours d'eau " (loc. cit.). Ce changement de préoccupation n'est pas sans incidence sur la gestion de l'eau. Désormais, elle ne se fait plus principalement en fonction de son 
usage, mais se fait en vue de sa protection. Comprendre ce changement de conception, du regard porté sur l'eau est crucial. La politique de l'eau actuelle, intégrant davantage ce caractère protecteur, est essentiellement issue de trois lois : la loi sur l'eau du 16 décembre 1964, la loi sur l'eau du 3 janvier 1992 et la loi sur l'eau et les milieux aquatiques (LEMA) du 30 décembre 2006. La réglementation française sur l'eau dépend aussi des directives européennes, traduites en droit français, notamment de la Directive Cadre de l'Eau (DCE) de 2000. Notons que les normes environnementales de l'Union Européenne déterminent aujourd'hui $80 \%$ de la législation française en matière environnementale (rpfrance.eu).

9 Du fait de l'interprétation et de l'application des normes européennes, les cours d'eau sont effectivement de plus en plus protégés. Leur qualité ainsi que leur masse et les masses (faune, flore, sédiments) y circulant doivent retrouver un équilibre dit naturel (DCE), sans gêne anthropique et idéalement, sans présence ni action anthropique. On parle de continuité écologique. Les aménagements à proximité d'un cours d'eau, sur celui-ci ou le bordant doivent donc prendre en considération les critères de protection des cours d'eau pour ne pas être accusés de nuire à l'objectif d'atteinte du bon état écologique.

\section{B - La quête : qu'est-ce qu'un cours d'eau?}

10 Afin que les aménagements sur ou à proximité d'un fossé répondent à la réglementation en vigueur, la première et néanmoins cruciale question à se poser est de savoir si l'on est en présence d'un cours d'eau. Ainsi, que reconnait-on comme cours d'eau et quels sont les dispositifs de gestion et de protection dont nous disposons alors? En l'occurrence, il s'agit de faire appliquer ou non les mesures de police définies par exemple par le code de l'environnement. Cependant, aussi cruciale que soit cette question, la législation française ne donne aucune définition de ce que peut être un cours d'eau. Sa qualification ne repose que sur la jurisprudence, construction prétorienne, construite au gré des cas spécifiques rencontrés. Preuve en est la circulaire récente - du moins récente au regard de l'ancienneté de la politique de l'eau - du 2 mars 2005, signée du Ministère chargé de l'Environnement, précisant la détermination d'un cours d'eau. Ainsi, un fossé est un cours d'eau si et seulement si sont attestées :

11 - La présence et la permanence d'un lit naturel à l'origine ;

12 - La permanence d'un débit suffisant une majeure partie de l'année.

13 La pertinence de ces critères a été confirmée par le Conseil d'Etat en 2011 (Arrêt EARL Cintrat du 21 octobre 2011), conférant à cette circulaire une force juridique et signant une définition de la notion de cours d'eau désormais jurisprudentielle. Notons que dans cet arrêt, l'alimentation par une source a également été mis en évidence comme critère attestant la présence d'un cours d'eau.

14 Sauf à connaître tous les fossés des hydrosystèmes, il semble impossible de vérifier ces critères par de simples visites ponctuelles. Des indices ont donc été choisis pour permettre la détermination des cours d'eau, notamment en cas d'absence d'eau: la présence de squelettes d'invertébrés aquatiques et/ou de plantes hydrophiles. Un schéma en arborescence reprend ces critères et indices en livrant l'interprétation qui doit en être faite (Fig. 2). 
Figure 2 : Tableau synthétique des critères de détermination et interprétation ${ }^{6}$

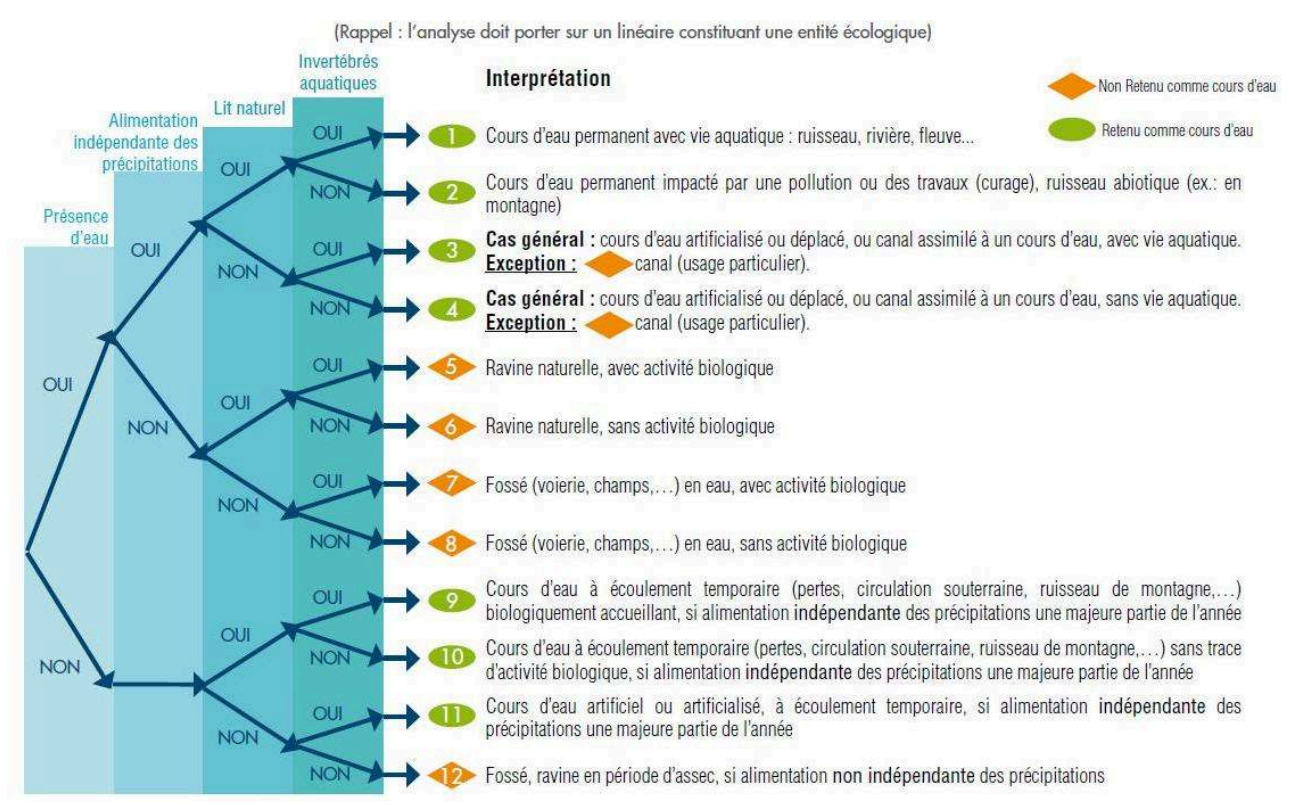

15 Pour donner une définition généraliste des géographes pour le cours d'eau, c'est un «terme générique pour tout élément du réseau hydrographique, tel que fleuve, rivière, ruisseau, ru, torrent; on parle de cours d'eau dès lors qu'existe un écoulement permanent, ou relativement stable dans ses intermittences " (Brunet et al., 2005, p.134). Les cours d'eau ont aussi un rôle ménagé qui leur est associé "ils "arrosent", « irriguent », « lavent » " (loc. cit.). Si l'on regarde la définition du ruisseau, c'est « un petit cours d'eau plus ou moins permanent». On note donc que la vision scientifique n'apporte pas en l'état des éléments supplémentaires permettant de mieux définir les cours d'eau.

\section{II - Application complexe de la notion de cours d'eau et incidences pour les étangs}

\section{A - Des clés de détermination complexes à appliquer}

Bien que reprises par tous les sites des services déconcentrés de l'Etat, aisément identifiables et citées par les agents, ces clés de détermination n'en restent pas moins vagues, nécessitant régulièrement l'appréciation d'un juge et étant souvent contestées par les personnes en lien avec le dit cours d'eau. La place à «l'interprétation » et le terme de "subjectivité » de la définition sont d'ailleurs repris dans les textes des services de l'Etat ${ }^{7}$ et des chercheurs ${ }^{8}$. Or, la définition d'un objet doit correspondre aux réalités de celui-ci. Pour comprendre la complexité de la détermination, il faut se pencher sur des exemples concrets.

Concernant la présence et la permanence d'un lit naturel à l'origine, deux situations sont possibles. Soit le fossé est resté naturel et il est identifiable par observation. Soit le fossé est artificialisé et il est nécessaire de recourir à des documents d'archives et à des témoignages pouvant attester la présence d'un ancien lit naturel modifié. Les cartes IGN permettent aussi d'attester la présomption d'existence d'un 
cours d'eau, donc d'un lit naturel à l'origine. A première vue, il paraît aisé de reconnaître l'existence de ce dernier. Outre la complexité de trouver des documents indiquant la présence d'un lit naturel à l'origine et la complexité de déterminer leur véracité, il est au préalable nécessaire de déterminer ce que l'on reconnaît comme un « lit naturel ». D'après la nature des preuves demandées, celui-ci doit être originel et non creusé par l'homme. Au regard de ce critère, une dérivation d'un cours d'eau ne peut être considéré comme un lit naturel. Pour autant, des classifications tendent à prouver le contraire. Pour exemple, l'ONEMA considère comme un cours d'eau le canal de dérivation du ruisseau de la Croulaie, affluent de La Nère (Cher), créé lors de la création de l'étang (Millot, 2015). Effectivement, les échanges suites à un procès verbal font bien mention de travaux réalisés dans le cours d'eau. Dès lors, soit un lit naturel peut être juridiquement créé, dans quel cas la notion de «naturel » semble toute relative, soit l'obligation d'un lit naturel ne l'est pas dans les faits, ce qui rend les critères de détermination pas moins flexibles. Malgré l'emploi d'une notion hydrologique, les appréciations juridiques semblent différentes et leur crédit supérieur. Ces deux conceptions pouvaient être à l'origine de conflits juridiques, du moins jusqu'à récemment. En effet, une instruction du gouvernement du 3 juin 2015 demandant autant que faire ce peut l'établissement d'une cartographie complète des cours d'eau donne les éléments de cadrage et de méthode afin d'aider aux cas complexes. A propos de l'existence d'un lit naturel à l'origine, elle stipule que «ce critère ne doit pas par ailleurs faire perdre de vue que, en fonction des usages locaux, des bras artificiels (tels que des biefs) laissés à l'abandon et en voie de renaturation peuvent être considérés comme des cours d'eau, [et ajoute également que] si un bras artificiel capte la majeure partie du débit, au détriment du bras naturel (et remettant en cause le critère de permanence de l'écoulement) le bras artificiel pourra être considéré comme cours l'eau » (MEDDE, 2015, p.6). La flexibilité de fait concernant la nécessité d'un lit naturel à l'origine est désormais institutionnalisée.

18 Concernant la permanence d'un débit suffisant une majeure partie de l'année, il n'y a pas de valeurs chiffrées. Le débit est jugé suffisant dès lors qu'il permet la présence d'une végétation hydrophile et d'invertébrés d'eau douce au moins six mois de l'année. Il peut donc aussi bien s'agir d'un filet d'eau que de flaques présentes dans le fond d'un lit, indépendantes des précipitations. L'absence de vie piscicole ne fait pas obstacle à cet indice. Comme le reprend un billet des agriculteurs de France : « difficile de ne pas être un cours d'eau!» (s.n., 2011 a). En effet, cette situation est bien évidemment le cas pour biens des fossés, pour exemple, des fossés de drainage. Effectivement, le vent emporte les graines des plantes hydrophiles, les oiseaux prennent dans leurs pattes, palmes, plumes divers graines, œufs ou larves et les emportent malgré eux, notamment dans les endroits où ils s'abreuvent. Ainsi, il peut aisément y avoir présence d'eau, de faune aquatique et de flore hydrophile. Bien que la première clé de détermination ne soit pas vérifiée (présence d'un lit naturel à l'origine) dans le cas d'un fossé de drainage, il arrive que certains soient classés comme cours d'eau, avec la réglementation qui s'ensuit. Plus encore, cette notion de " débit suffisant une majeure partie de l'année " implique directement l'existence d'autres débits insuffisants une majeure partie de l'année. Pour autant, loin de ne pas être un cours d'eau, ce type de fossé est tout simplement classé comme cours d'eau temporaire. Ce fait, ajouté au constat du tableau synthétique des critères de détermination et interprétation, semblerait indiquer que le critère de débit suffisant n'est pas réellement un critère déterminant dans la définition d'un cours d'eau, mais plutôt dans la 
qualification du type de cours d'eau. Pour autant, à l'heure actuelle, la réglementation qui s'applique à ces deux types de cours d'eau est identique. Cette situation peut poser un problème d'efficience des aménagements induits pour les étangs. Cela s'avère être le cas par exemple pour l'étang du Moulin, situé sur la commune de Chaillac (Indre). Cet étang, présent sur la carte de Cassini (1750) était en projet de restauration et de remise en eau en 1998. Après des années d'études, de courriers et de procès, L'Allemette a bien été reconnue comme cours d'eau non dépendant des eaux pluviales, au moins $1 \mathrm{~km}$ en amont de l'étang, et ce, malgré plusieurs avis précédents contradictoires. La réalisation d'une dérivation a été obligatoire, même après l'ouverture de l'étang et malgré l'avis positif du document d'incidence initial sur l'impact de l'étang. Ce que l'on constate aujourd'hui, c'est que la dérivation est à sec de manière générale entre juillet et septembre. $\mathrm{Si}$, comme les premières études le pressentent ${ }^{9}$, la dérivation est essentiellement utile en été, celle de l'étang du moulin permet de répondre aux exigences réglementaires, sans pour autant améliorer l'impact thermique ou la continuité écologique en général.

19 L'analyse des clés de détermination tend à montrer que le critère de détermination principal serait en réalité la dépendance totale ou non de l'écoulement aux précipitations.

\section{B - Des outils d'aide à la détermination efficients ?}

Nous avons vu que certains documents peuvent aider à la détermination des cours d'eau. Notamment, les cartes IGN sont un outil privilégié par les agents en charge de la police de l'eau. La cartographie des cours d'eau, permanents ou non, est effectivement une aide précieuse, permettant d'obtenir une réponse rapide et facile d'accès. Toutefois, toute personne connaissant bien un réseau hydrographique aura noté sur ces cartes à la fois les absences de certains cours d'eau, la mauvaise localisation d'autres ou encore la présence de cours d'eau inexistants sur le terrain. Bien que le terme de «présomption d'existence » soit employé au sujet d'un cours d'eau répertorié par l'IGN (ce qui n'est toutefois pas toujours le cas), cette information reste une preuve solide dans le cas d'un litige. Un fossé répertorié comme cours d'eau par l'IGN reste très difficile à déclasser. L'absence régulière du mot " présomption » dans les textes en lien avec le sujet traduit par ailleurs le poids important de cette source aux yeux de tous. Considérant les failles des informations IGN, cet outil devrait plus encore être utilisé à sa juste valeur.

21 En cas de doute sur la détermination d'un cours d'eau, il convient également de contacter le service départemental chargé de la police de l'eau. Il existe deux cas de figure : le fossé en question est bien connu des agents et ceux-ci peuvent déterminer en connaissance de cause l'existence du cours d'eau - selon les critères vus précédemment - ou les agents ne connaissent pas le fossé et la détermination se fera après expertise. Le jugement se fait alors à par un constat immédiat, en fonction des preuves visibles : talweg, berges d'au moins $10 \mathrm{~cm}$, vie aquatique. Ces preuves doivent permettre d'établir une détermination rapide par les agents chargés de la police de l'eau. Toutefois, si l'appréciation est possible, elle ne reste le fait que d'une visite. Le jugement peut malheureusement s'appuyer sur des preuves erronées, comme la présence ponctuelle de batraciens. 
Pour pallier les problèmes liés à l'identification des cours d'eau, l'instruction du gouvernement du 3 juin 2015 demande l'établissement d'une cartographie complète des cours d'eau. Pourtant, elle laisse toute la largeur et le flou nécessaires dans l'exécution de la tâche ${ }^{10}$ comme dans les éléments de cadrage qui reprennent les critères existants (Agents du Service Eau et Risques, 2011, pp.15-16), tout en prenant comme «base à une cartographie exhaustive » (loc. cit., p.5) les référentiels cartographiques dont on connait les failles. Ce faisant, il ne semble pas que cette cartographie doive changer la donne quant aux difficultés énoncées.

\section{C - La distinction des cours d'eau par typologie}

23 Les cours d'eau continentaux n'ont pas les mêmes caractéristiques : géologie, climat, débit, végétation, espèces aquatiques etc. Géographes et gestionnaires de l'eau ont ainsi créé des typologies de cours d'eau, afin d'en identifier rapidement les caractéristiques. Il existe plusieurs hiérarchisations du réseau hydrographique (Strahler, Horton, Gravelius, Shreve). L'ONEMA a réalisé une étude (ONEMA, 2010 b) sur les typologies de cours d'eau et leurs caractéristiques correspondantes. S'il n'existe pas de réglementation distincte pour la protection des cours d'eau temporaires et permanents, il existe une réelle interrogation sur la distinction des cours d'eau afin d'en adapter la gestion. La typologie "masse d'eau » de la DCE a elle-même pris en compte le rang de Strahler, afin d'identifier des secteurs homogènes (Fig. 3).

Figure 3 : Principe de l'ordination de Strahler (onema.fr)

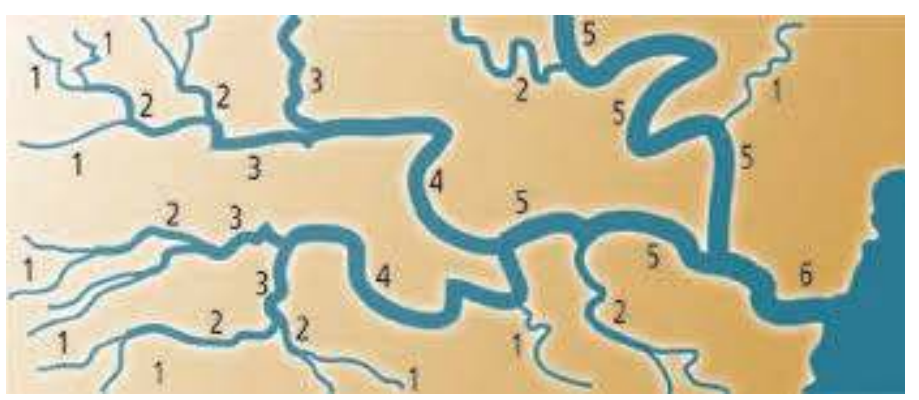

Six classes de taille ont ainsi été définies: très petits, petits, moyens, grands, très grands cours d'eau et grands fleuves. La zonation longitudinale de la typologie de Strahler consiste à prendre en compte le tronçon comme unité hydrographique. Chaque tronçon d'ordre $\mathrm{n}+1$ est issu de la confluence de deux tronçons d'ordre $\mathrm{n}$. Un tronçon n'ayant pas d'affluent se voit attribuer le rang 1. La seconde distinction considérée pour créer la typologie de la DCE est celle d'hydro-écorégion, créée par le Cemagref en 2002. En plus d'un découpage longitudinal, on considère ici les caractéristiques déterminant le fonctionnement de l'écosystème hydrologique: géologie, relief et climat. «Le principal enjeu de cette typologie concerne la définition des conditions de référence à partir desquelles seront établis les états écologiques » (Malavoi et Bravard, 2010). Deux autres méthodes de distinction sont utilisées: la typologie géodynamique fonctionnelle, utilisée lors de diagnostics hydromorphologiques, et la zonation écologique, permettant de constater la perturbation d'un milieu en fonction de son peuplement, d'après la répartition théorique des espèces en absence de perturbation (loc. cit.). 


\section{D- Cours d'eau et étangs, entre réglementation, protection et aménagements : une compatibilité possible?}

La perception de la ressource en eau est aujourd'hui environnementale. L'objectif affiché par les services administratifs et l'Union Européenne est de restaurer, du moins améliorer, sa qualité. Le cœur de cette ressource continentale est le cours d'eau. Notre interrogation consistait à déterminer à quel moment nous sommes en présence d'un cours d'eau. Dans un premier temps, la lecture de la jurisprudence et de son détail semble indiquer que la majorité des indices permettant la détermination d'un cours d'eau doit être rassemblée. Mais on constate régulièrement que la seule présence d'un de ces indices suffit à la détermination.

Il est vrai que la définition à la fois large et subjective du cours d'eau permet au législateur de définir nombres de fossés comme cours d'eau. Plusieurs facteurs expliquent cette situation. La vision environnementaliste et juridique d'objets géographiques ne permet pas la prise en compte de leurs spécificités. L'absence d'autres définitions possibles pousse au recours à la notion de cours d'eau, solution de classification. La typologie elle-même se construit une fois les fossés classés en cours d'eau, et non en amont, pour distinguer les différents types de ressource en eau. Cette notion de cours d'eau offre aux services déconcentrés en charge de la protection de l'eau un régime réglementaire fort leur permettant d'agir au mieux pour répondre aux critères de bon état écologique des masses d'eau ordonnés par l'Union Européenne. Dès lors, il est aisé de comprendre son utilisation.

La volonté de protéger la ressource en eau n'est pas l'objet de la critique, loin de là. La protéger est une noble cause : économique, philosophique, sociale. Mais la combinaison d'objets de natures différentes aux fonctionnements propres soumis à une réglementation identique peut entraîner une protection inadaptée, voire néfaste. Si tout ou presque est cours d'eau et que l'application de la réglementation qui en découle est obligatoire mais peut être inadaptée, la solution est peut-être de prendre en compte les particularités des cours d'eau (rang, permanence) et d'y associer une réglementation différenciée. Par exemple, la dérivation de l'étang du moulin à Chaillac a été imposée mais est pour sûr inefficace une partie de l'année du fait de l'assèchement du cours d'eau. On pourrait donc penser qu'il ne soit pas possible d'imposer une dérivation dans le but d'améliorer la qualité de l'eau lorsque le cours d'eau est temporaire. L'idée de cette distinction est de faire en sorte que la protection des cours d'eau et l'aménagement des étangs soient compatibles et efficaces.

A l'heure actuelle, l'aménagement souhaité est la dérivation, qui doit réduire les méfaits des étangs généralement portés comme suit :

- Rupture de la continuité écologique et arrêt du «transport naturel des sédiments de l'amont vers l'aval d'un bassin versant et la circulation des espèces piscicoles" (ONEMA, 2013)

- Réchauffement de l'eau et modification de sa qualité physico-chimique

- Pollution aval des cours d'eau par colmatage des habitats/frayères lors des vidanges

- «Impacts importants [...] sur la qualité et la quantité de la ressource en eau et sur les milieux aquatiques » (loc. cit.)

Avant de promouvoir, voire d'imposer ce type d'aménagement, il faut comprendre et quantifier ses différents impacts. Ceux-ci concernent tout à la fois l'aspect physique du 
milieu (cours d'eau amont et aval, fonctionnement interne de l'étang), l'aspect usuel, dépendant en partie du premier aspect ainsi que des modifications statutaires possibles, et l'aspect économique, dépendant en partie du second aspect ainsi que du coût des travaux et entretiens.

\section{III - Conséquences de la réglementation des cours d'eau sur l'aménagement des étangs : la dérivation, une " solution » pérenne?}

La dérivation représente $8,1 \%$ des modes d'alimentation des étangs sur l'ensemble du Limousin et 12,4 \% dans le bassin de la Vauvre (Boischaut). C'est donc un aménagement aujourd'hui peu répandu mais qui est "amené à se répandre dans les années qui viennent » (Touchart et Bartout, 2011). Le principe de la dérivation n'est pas récent et reprend celui du bief des moulins. La différence avec son utilisation passée réside dans l'intérêt porté à cette technique. Essentiellement d'usage commercial et pratique gestion des débits pour la production d'hydro-électricité, gestion d'étangs piscicoles, apport d'eau potable aux habitations - la dérivation est désormais le moyen de répondre à des objectifs environnementaux. De l'ordre du théorique, cette idée doit être vérifiée, et ce, à différentes échelles spatio-temporelles. Mais nous n'aurons alors que l'aspect physique de la chose. Il subsiste un aspect tout aussi crucial à exposer : l'aspect financier.

\section{A - Dérivation et continuités : un atout essentiellement estival}

La continuité écologique est un point essentiel de convergence entre la DCE, la trame verte et bleue (loi Grenelle 1 du 3 août 2009) et la loi sur l'eau de décembre 2006. Lorsque l'ONEMA recense plus de $60000^{11}$ seuils et barragesen France (Weingertner et Roussel, 2010) nuisant à la continuité écologique, il va sans dire que les étangs sont aussi concernés. La dérivation est officiellement valorisée pour garantir cette continuité écologique. Or, le chenal de dérivation étant à proximité des étangs, celui-ci doit immanquablement franchir la digue. Ainsi, soit nous sommes en présence d'une pente très forte que les poissons ne pourront pas franchir, soit nous sommes en présence d'une pente douce où se trouvera un seuil infranchissable lui aussi (Tableau 1).

Tableau 1 : Capacité de franchissement des seuils par les truites (s.n., 2010 a)

\begin{tabular}{|c|c|}
\hline Hauteur de chute & Passage de la truite \\
\hline$<20 \mathrm{~cm}$ & ok \\
\hline $20-40 \mathrm{~cm}$ & délicat \\
\hline $40-60 \mathrm{~cm}$ & très difficile \\
\hline$>60 \mathrm{~cm}$ & impossible \\
\hline
\end{tabular}

Il n'est donc pas exact que la dérivation à elle seule est la meilleure solution pour garantir la continuité écologique. 

l'eau déterminant la qualité de l'eau des cours d'eau et la santé de la faune et de la flore, elle est un atout dans l'étude visant à préciser l'utilité de la dérivation. C'est pourquoi il est intéressant d'étudier le bilan thermique amont/aval des étangs à dérivation (Millot, 2015). L'étude la plus probante serait évidemment celle d'un étang avant et après sa mise en dérivation. Les circonstances ne s'y étant pas encore prêtées et les données existantes étant peu nombreuses (Bartout et Touchart, 2013), nous pouvons nous baser sur des études préalables (Touchart, 2007) menées sur le bilan thermique des étangs en fonction des types de sortie d'eau. Dans l'objectif d'avoir ce qui pourrait s'apparenter à l'optimum de l'utilité de la dérivation, nous n'allons pas présenter les bilans de différents sites d'étangs que nous avons étudiés mais le bilan d'un étang à dérivation relevant presque du prototypage.

L'étang en question est un étang d'agrément privé, localisé à Montchevrier (Indre), nommé «Etang de Gâte-Souris». Ancien étang servant à alimenter un moulin (représenté sur la carte de Cassini), il barre le cours d'eau permanent La Bouzanne, de rang 1 (Strahler) à cet endroit. Premier étang de cette tête de bassin, il mesure approximativement 2 ha (Fig. 4), auxquels s'ajoute un premier petit étang à usage de rétention des sédiments d'une surface de 0,5 ha. Il dispose d'un déversoir de surface, nettoyé quotidiennement. La dérivation a été construite en 2008, suite à l'acquisition de l'étang par l'actuel propriétaire et aux travaux de restauration (vidange, curage, restauration de la queue et des berges). Creusée à même la terre et la roche granitique en son bout, elle parcourt les 300 mètres séparant le répartiteur, situé à proximité directe du premier étang, et le déversoir de surface, sans autre affluent. Ses dimensions moyennes sont de un mètre de large pour un mètre de profondeur. La hauteur d'eau, selon les endroits et la saison, se situe entre 10 centimètres et 40 centimètres.

Figure 4 : Schéma de l'étang Gâte-Souris (Montchevrier)

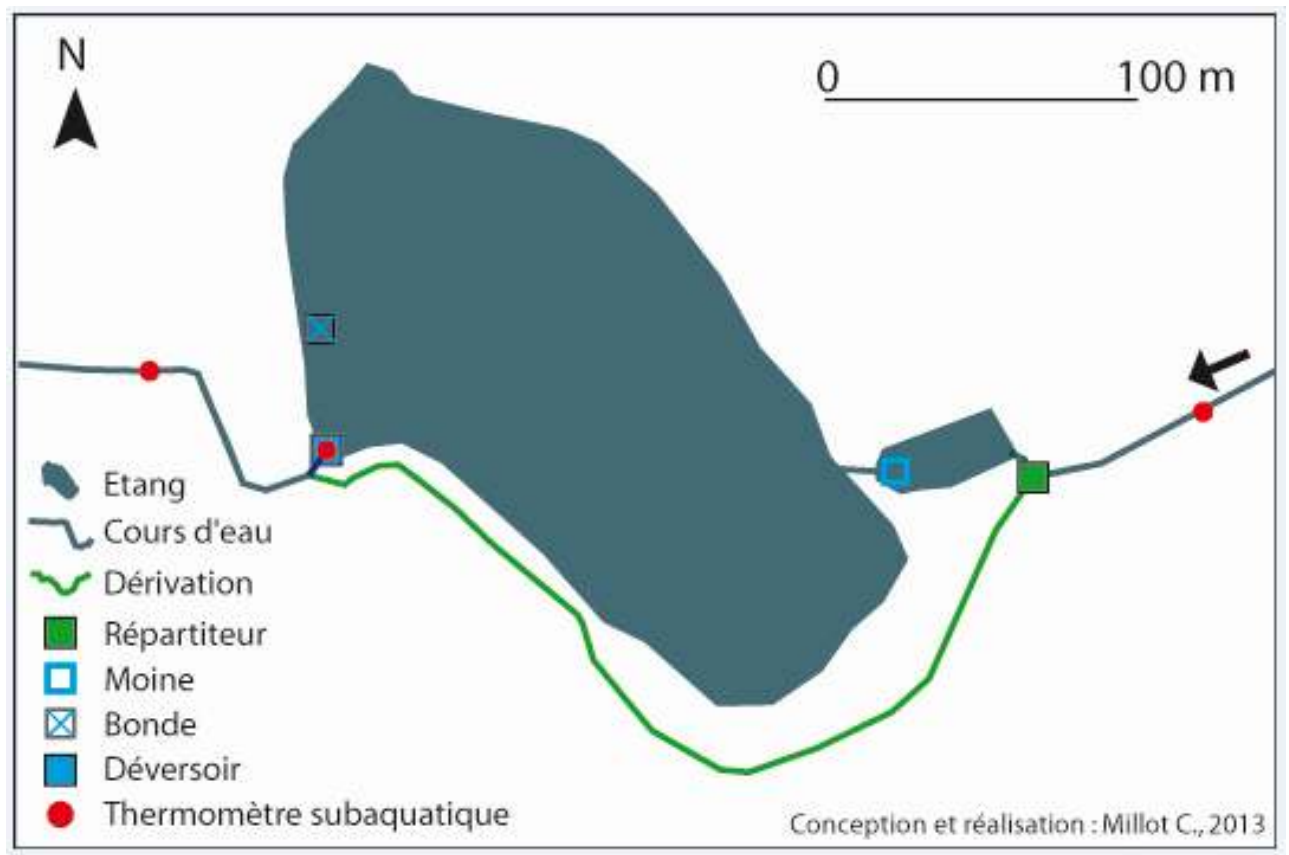

Le matériel utilisé pour mesurer les températures est trois thermomètres enregistreurs subaquatiques. Ce sont des Tinytag Datta Loggers dont la sonde interne, qui a un temps 
de réponse d'une minute et demie, est protégée par un boîtier IP-68 résistant aux chocs et étanche jusqu'à $15 \mathrm{~m}$ de profondeur ${ }^{12}$. Les thermomètres sont programmés pour prendre une mesure par heure. «Ces instruments ont l'inconvénient d'une précision thermique médiocre, puisque ce sont des thermomètres à sonde piézoélectrique » (Touchart et Bartout, 2011, p.151). Cependant, les thermomètres utilisés lors de l'étude étaient neufs et leur précision atteignait donc un dixième de degré (loc. cit.). Ils ont été placés comme suit : 50 mètres en amont du répartiteur, au déversoir de surface - côté étang - et 50 mètres en aval de la jonction entre l'exutoire et la dérivation.

L'étude sur une année entière, du 20 avril 2012 au 19 avril 2013, a permis l'enregistrement de plus de 26000 données inédites, le tout réparti entre l'amont de l'étang, son déversoir et l'aval. Les données du thermomètre aval comprises entre le 13 février et le 16 mars ont été exclues du traitement parce qu'elles ne sont pas fiables. Pour exemple, le 15 mars 2013 , le thermomètre a enregistré $-3,10^{\circ} \mathrm{C}$ à $8 \mathrm{~h}$ et $20,34^{\circ} \mathrm{C}$ à 13h. Nous pensons que durant cette période, des poches de glace se sont formées ou se sont mises contre l'appareil et l'ont déréglé. Ainsi, les journées indiquant à la fois de l'eau en surfusion puis de l'eau dépassant $10^{\circ} \mathrm{C}$, ce qui est le cas pour toutes les journées durant cette période, et exclusivement pour cette période, n'ont pas été prises en compte pour les trois points de mesures (2 301 données). L'absence d'un mois de températures hivernales (froides) a pour effet une légère surévaluation de la moyenne annuelle de température. Cependant, les tendances restent inchangées avec les 23976 températures restantes.

D'après les données, on peut affirmer qu'à l'échelle de l'année, la continuité thermique entre l'amont et l'aval est assurée. En effet, on mesure un réchauffement de $0,35^{\circ} \mathrm{C}$, ce qui est négligeable. Cette continuité thermique semble avoir été permise par la dérivation puisque la moyenne annuelle des températures au déversoir indique $2,93^{\circ} \mathrm{C}$ de plus qu'à l'amont. Plus précisément, entre le 20 avril 2012 et le 19 avril 2013, la température moyenne de l'eau en amont de l'étang, est de $11,69^{\circ} \mathrm{C}$. Elle est de $14,62^{\circ} \mathrm{C}$ à la sortie du déversoir et de $12,04^{\circ} \mathrm{C}$ en aval (Fig. 5).

Figure 5 : Moyennes annuelles de températures en amont, au déversoir et à l'aval de l'étang GâteSouris (20 avril $2012-19$ avril 2013)

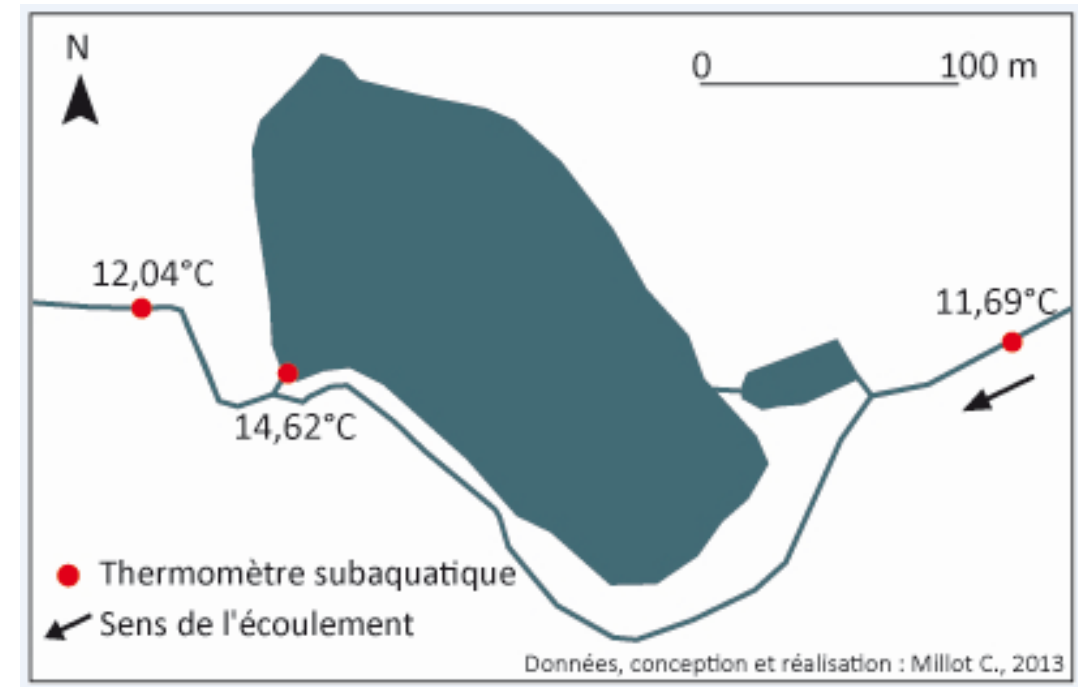

De manière plus générale, l'augmentation de la température à la sortie d'un étang à déversoir est de $2^{\circ} \mathrm{C}$ (Touchart, 2007, p.138). Il faut toutefois préciser qu'au-delà de la 
légère surévaluation, du fait du manque d'un mois de températures hivernales, la tranche d'eau au niveau du déversoir est très petite : 20 centimètres au plus. De plus, cette pellicule n'est pas protégée par la végétation. Elle est donc très influencée par la température extérieure, le vent et l'exposition solaire. Ceci peut expliquer le réchauffement moyen annuel plus important que la moyenne.

A première vue, cet étang à dérivation n'a pas d'impact sur le cours d'eau aval. L'aménagement en dérivation semble utile au rétablissement thermique de l'eau sortant de l'étang. Evidemment, cette analyse est intéressante pour avoir un point de vue général sur l'impact thermique d'un étang à dérivation mais il faut nuancer ces résultats par une étude à plus grande échelle temporelle, de l'ordre de la saison, du mois et même du jour. En effet, ces échelles, tout en restant générales, sont plus intéressantes pour comprendre l'impact sur la vie aquatique (Tableau 2).

Tableau 2 : Synthèse du réchauffement thermique par l'étang Gâte-Souris ${ }^{13}$ (20 avril $2012-19$ avril 2013)

\begin{tabular}{|l|c|c|}
\hline & Bilan déversoir-amont & Bilan aval-amont \\
\hline Moyenne annuelle de réchauffement & 2,93 & 0,35 \\
\hline Moyenne estivale & 7,88 & 2,96 \\
\hline $\begin{array}{l}\text { Moyenne mensuelle du plus fort réchauffement } \\
\text { déversoir-amont }\end{array}$ & 8,1 & 3,57 \\
\hline $\begin{array}{l}\text { Moyenne de la journée du plus fort réchauffement } \\
\text { déversoir-amont }\end{array}$ & 10,41 & 7,52 \\
\hline Moyenne hivernale & $-3,42$ & $-3,12$ \\
\hline $\begin{array}{l}\text { Moyenne de la journée du plus fort refroidissement } \\
\text { déversoir-amont }\end{array}$ & $-5,65$ & $-4,76$ \\
\hline
\end{tabular}

Durant l'été, comme durant le mois où la moyenne des températures de l'eau à la sortie de l'étang est la plus chaude par rapport à celle en amont, la dérivation permet là encore de réduire cette augmentation thermique. Si la récupération thermique se produit naturellement en aval (Touchart, 2007), on peut aisément supposer que pour cet étang, sans ce système de dérivation, elle ne serait pas aussi importante 50 mètres seulement après le déversoir. La quantification précise nécessiterait une comparaison avec un autre étang similaire sans dérivation. Mais ce que l'on peut avancer, c'est que la dérivation semble diminuer la distance et le temps nécessaire à la récupération thermique de cet étang en été, même lors des plus forts réchauffements. Cependant, si la réduction de la température par la dérivation est plus importante en été - l'eau en aval est en moyenne $20 \%$ plus froide que l'eau sortant du déversoir contre $17,6 \%$ en moyenne sur l'année - la continuité thermique est davantage perturbée puisque nous avons une augmentation de presque $3^{\circ} \mathrm{C}$. C'est un degré de moins que le réchauffement moyen par un étang à déversoir et identique à celui d'un étang à moine (Touchart, 2007). De la même manière, la moyenne mensuelle du plus fort réchauffement en aval est quasiment identique à celle d'un étang muni d'un moine, qui est de $4^{\circ} \mathrm{C}$ (Touchart, 2007).

En hiver, la température moyenne de l'eau sortant du déversoir est particulièrement plus froide que celle en amont. Là encore, cela est dû aux caractéristiques du déversoir étudié. Dans ce cas, la dérivation n'atténue que très légèrement le phénomène $\left(+0,3^{\circ} \mathrm{C}\right)$.

Si à l'année, l'étang à dérivation apparait comme non impactant et plus avantageux qu'un étang sans dérivation muni d'un déversoir ou d'un moine, l'étude multi-scalaire est moins idéale. En été, l'aménagement réduit l'impact de l'étang à déversoir, mais pas plus qu'un étang qui serait muni d'un moine. En hiver, l'intérêt est quasi nul. Mais si 
l'on reprend en compte l'impossibilité de mettre un moine (hauteur d'eau insuffisante) et l'impact particulièrement négatif du déversoir (pellicule d'eau fortement exposée), on peut conclure que l'aménagement en dérivation est une solution adaptée et efficace pour réduire les réchauffements estivaux de ce type d'étangs.

\section{B - Usage, économie, coût et entretien : vers un effacement programmé ?}

Si intéressante que puisse être la dérivation d'un point de vue écologique, celle-ci devrait être économiquement réalisable et supportable pour exister, que ce soit par un coût raisonnable ou par l'intérêt économique qu'elle présente pour rentabiliser l'investissement.

La création d'une dérivation n'est pas à moindre coût. Un exemple de travaux sur trois étangs du bassin du Cousin en Bourgogne en donne un aperçu. Le coût de l'étude de faisabilité est chiffré à 7000 euros tandis que les travaux de contournement d'un des étangs sont chiffrés à 11308 euros (ONEMA, 2010 a). En plus des études et du coût des travaux, il peut éventuellement y avoir le coût de l'acquisition de parcelles. Dans ce cas précis, le montant des travaux a été pris en charge par des financements publics dans le cadre du programme Life Nature "Ruisseaux de têtes de bassins et faune patrimoniale associée". D'autres projets sont ainsi financés pour tout ou partie après signature de conventions avec les propriétaires ou syndicats de propriétaires, avec les contributions des Agences de l'Eau ou de l'UE via le FEDER (s.n., 2012).

Toutefois, ces cas de financement restent des exceptions parmi les 554566 plans d'eau en France (Bartout et Touchart, 2013). La dérivation peut être imposée et sauf jugement favorable, les propriétaires doivent aménager leur étang. Celui-ci est certes privé, mais l'investissement imposé doit avoir une rentabilité publique (bon état écologique). Il est intéressant de se poser cette question : doit-on considérer que le coût du dit mauvais état écologique des cours d'eau doit être pris à la seule charge des particuliers ? La situation semble montrer que les autorités en charge de la gestion de l'eau ont tranché cette question : le particulier doit assumer l'aménagement de son bien pour répondre à la réglementation. Et pourtant l'effacement de ce même bien privé, lui aussi coûteux ${ }^{14}$, est davantage aidé voire pris en charge. Tout cela reste dépendant des régions et des Agences de l'Eau. Par exemple, la région Limousin finance à hauteur de $30 \%$ maximum un effacement, contre $20 \%$ maximum pour des travaux de continuité (Munoz et FaviAuguste, 2012). D'autres régions ne financent pas ce genre de projets.

$50 \mathrm{Au}$ vu des nombreuses contraintes financières des étangs (études pour leur création, pour leur vidange, aménagements multiples, entretien des lieux etc.), la valorisation économique est cruciale. En ce sens, la dérivation peut être intéressante car une fois l'étang déconnecté du cours d'eau, celui-ci devient eau close. La pisciculture et la mise en valeur de l'étang est ainsi possible (Bachasson, 1997 ; Schlumberger et Girard, 2013). Toutefois, bien des étangs n'ont pas vocation à être utilisés pour la pêche. Ainsi s'explique le premier argument de la fiche technique expliquant l'intérêt de l'effacement d'un étang: la "suppression des coûts d'entretien» (s.n., 2010 b). L'ONEMA précise aussi que "l'effacement d'un ouvrage hydraulique autorisé peut être envisagé, à la demande du propriétaire, lorsque les frais d'entretien et de mise aux normes de l'ouvrage excèdent l'utilité de son maintien" (Weingertner et Roussel, 2010). Plus généralement, il est dit que « la solution de l'effacement est à préconiser 
pour les ouvrages aujourd'hui abandonnés, sans usage ou sans intérêt, qu'il soit économique, patrimonial ou paysager. Cette option présente en effet beaucoup d'avantages en termes de rétablissement complet de la continuité écologique et de simplicité de gestion par la suite» (loc. cit.). Cet effacement "peut également être envisagé par le préfet lorsque ces ouvrages hydrauliques sont incompatibles avec les enjeux environnementaux et notamment les engagements de la France relatifs à la législation européenne » (loc. cit.). En considérant ces propos, l'absence régulière de financement de la dérivation - face au financement de l'effacement - pourrait-elle entre autre être perçue comme une situation de stress financier créée, visant au non respect involontaire de la réglementation et/ou à l'abandon de l'étang, menant à sa suppression?

\section{Conclusion}

51 A la question de savoir les raisons pour lesquelles les étangs posent problème en France, on peut aisément dire que ce qui est en jeu, ce sont les impacts supposés des étangs qui seraient à l'encontre des objectifs du bon état écologique des cours d'eau. La première remarque à faire, c'est que ces impacts sont méconnus car en cours d'étude. On ne peut donc ni attester, ni nier de manière scientifique les impacts des différents types d'étangs. Il est toutefois possible de les relativiser au vu des autres usages et aménagements anthropiques en lien avec les cours d'eau tel que l'agriculture, les barrages hydroélectriques, ou la pollution industrielle des sols et cours d'eau.

La réglementation sur les cours d'eau est basée sur une « définition » de l'objet central qui est complexe. En effet, la notion de cours d'eau est une définition générale appliquée aux écoulements. A contrario, les clés de déterminations doivent donner des indices fiables et non discutables sur l'occurrence ou non d'un cours d'eau. Mais ces critères sont parfois eux-mêmes complexes, comme ce que doit être l'aspect « originel » d'un lit, et appliqués avec subjectivité. Ces clés ne permettent donc pas d'avoir une détermination qui fasse l'unanimité, quels que soient les usages et usagers. Si la détermination est complexe, les typologies de cours d'eau sont connues et reconnaissables. Il serait donc possible d'y appliquer une gestion différente dans le but de parvenir à une gestion optimale, réalisable et ne portant pas préjudice à tort aux aménagements existants. Le rang, le débit et la permanence du cours d'eau semblent être des critères intéressants dans la distinction des cours d'eau en vue de leur aménagement. Du moins cela se vérifie-t-il pour la dérivation d'après les exemples étudiés.

53 A l'heure actuelle, la dérivation est promue comme l'aménagement le plus efficace permettant de réduire les impacts négatifs des étangs. Elle est de plus en plus requise lors de création d'étangs, de travaux d'aménagement ou lors des ventes. L'étang de Gâte-Souris permet de comprendre ce que la dérivation peut offrir de mieux. D'après le bilan annuel des températures, on comprend que la dérivation peut être utile sur ce type d'étang. L'utilité se vérifie essentiellement en période estivale, tout comme pour un étang muni d'un moine fonctionnel. Désormais, il faut continuer l'étude de différents types d'étangs, à plus long terme et sur d'autres échelles spatiales pour connaître les conditions pour lesquelles la dérivation est efficiente.

Si la dérivation peut physiquement et écologiquement être utile, sa réalisation peut poser problème. On note effectivement que le coût engendré pose et posera un 
problème aux propriétaires. En conservant cette situation, il est certain que les propriétaires ne pourront pas toujours appliquer la réglementation et seront amenés à mettre à sec voire supprimer l'étang. Ceci est d'autant plus dommage si l'aménagement est demandé sans que son efficacité se vérifie par la suite. Si malgré son coût, la dérivation reste un aménagement souhaité, il est nécessaire de reconsidérer sa prise en charge. Car cette question du coût de la dérivation, aménagement préconisé au vu de la réglementation des cours d'eau et de l'impact supposé des étangs, met en jeu la pérennité des étangs.

\section{BIBLIOGRAPHY}

- Agence de l'Eau Seine-Normandie, 2006, Catalogues coûts et bénéfices unitaires, Catalogue, URL : http://www.documentation.eaufrance.fr/notice/catalogues-couts-et-benefices-unitaires0

- Agents du Service Eau et Risques, 2011, Dossier loi sur l'eau, mode d'emploi, Fiche technique, DDT du Gers, 40 p., consulté en janvier 2013, URL : http://www.gers.gouv.fr/content/download/ 6417/39470/file/Fiches_Integrale_juin2012.pdf

- Bachasson B., 1997, Mise en valeur des étangs, Paris, Technique et documentation, 176 p.

- Bartout P., Touchart L., 2013, « L'inventaire des plans d'eau français : un outil d'une meilleure gestion des eaux de surface », Annales de géographie, n691, p.266-289

- Berteaud P., 2005, Circulaire relative à la définition de la notion de cours d'eau, Directeur de l'eau pour le Ministre de l'écologie et du développement durable, 02/03/05

- Brunet R., Ferras R., Théry H., 2005, Les mots de la géographie, dictionnaire critique. MontpellierParis, RECLUS - La documentation Française, 520 p. (3e édition)

- FORGECO, 2010, La protection des cours d'eau et des zones humides, Fiche technique, Programme SYSTERRA, mis en ligne le 27 juillet 2010, consulté le 10 mai 2013, URL : https:// forgeco.cemagref.fr/reglementation-environnementale-et-exploitation-forestiere/fiche-nb015la-protection-des-cours-deau-et-des-zones-humides

- Malavoi J.R., Bravard J.P., 2010, Eléments d'hydromorphologie fluviale, Onema, 224 p., consulté le 18 juin 2013, URL : http://www.onema.fr/IMG/pdf/elements-dhydromorphologie-fluviale.pdf

- Marc Ph., 2006, Les cours d'eau et le droit, Paris, Editions Johanet, 292 p.

- Millot C., 2015, Les étangs à dérivation : une réponse aux nuisances environnementales ? Etude de sites en barrage de rangs $0,1,2$ et 4, en Indre, Cher et Creuse, Thèse, Université d'Orléans, 259 p.

- Ministre de l'écologie, du développement durable et de l'énergie, 2015, Instruction $d u$ Gouvernement du 3 juin 2015 relative à la cartographie et l'identification des cours d'eau et à leur entretien, Directive, 9 p., consulté en décembre 2015, URL : http://circulaire.legifrance.gouv.fr/pdf/ 2015/06/cir_39701.pdf 
- Munoz A., Favi-Auguste Ch., 2012, Les financements publics en faveur de l'amélioration de la gestion des étangs, Présentation, Région Limousin, 15 février 2012, consulté le 4 septembre 2013, URL : http://www.eptb-vienne.fr/IMG/pdf/11_C-FAVI_presentation_financement_etgs.pdf

- ONEMA, 2010 a, Suppression ou dérivation d'étangs sur cours d'eau, Fiche technique, consulté le 10 mars 2012, URL : http://www.onema.fr/IMG/Hydromorphologie/22_0_intro_r2_vbat.pdf

- ONEMA, 2010 b, Des étapes et des outils, Fiche technique, 39 p., consulté le 16 septembre 2013 , URL : http://www.onema.fr/IMG/Hydromorphologie/III_Etapes_et_outils.pdf

- ONEMA, 2013, Les actions de la Police de l'eau dans la gestion des plans d'eau, Dossier de presse, 26 p., consulté le 10 septembre 2013, URL : http://www.onema.fr/IMG/pdf/DOSSIER-DE-PRESSECOMPLET.pdf

- Parlement européen, Conseil de l'Union Européenne, 2000, La directive cadre européenne sur l'eau, Journal officiel des Communautés européennes, $72 \mathrm{p}$.

- Schlumberger O., Girard P., 2013, Mémento de pisciculture d'étang, 5è édition, Paris, Quae, 224 p.

- s.n., 2010 a, Mémento du pêcheur de truites : connaître, préserver le milieu et les poissons, Mémento, Fédération du Rhône pour la Pêche et la Protection des Milieux Aquatiques, 22 p., consulté le 20 mai 2013, URL : http://www.breche.fr/images/Patrimoine\%20piscicole/ memento_du_pecheur_de_truites.pdf

- s.n., 2010 b, Gestion des étangs, l'effacement, une solution à envisager, Fiche technique, EPTB Vienne, consulté en mai 2012, URL : http://www.eau-loire-bretagne.fr/les_rendez-vous_de_leau/ les_rencontres/rencontres_2010/6-Effacement_etang.pdf

- s.n., 2011 a, Il est difficile de ne pas être un cours d'eau !, Actualité juridique, Agriculteurs de France, consulté le 10 septembre 2013, URL : http://www.agriculteursdefrance.com/fr/

ContenuJuridique.asp?Num=759

- s.n., 2011 b, Notion de cours d'eau, guide pratique de détermination, Guide, Région Midi-Pyrénées, p. 13, consulté en juin 2012, URL : http://www.midi-pyrenees.developpement-durable.gouv.fr/IMG/ pdf/Notion-de-cours-eau_cle05c823.pdf

- s.n., 2012, Gestion des étangs, des travaux déjà terminés, Actualités, Syndicat d'aménagement du bassin de la Vienne, consulté le 10 mai 2013, URL : http://www.syndicat-bassin-vienne.fr/Accesdirects/Actualites/Gestion-des-etangs-des-premiers-travaux-deja-termines

- Touchart L. (dir), 2007, Géographie de l'étang : des théories globales aux pratiques locales, Paris, L'Harmattan, $228 \mathrm{p}$.

- Touchart L., 2007, « L'étang et la température de l'eau : un ensemble d'impacts géographiques », in Touchart L. (dir), Géographie de l'étang : des théories globales aux pratiques locales, Paris,

L'Harmattan, p.119-156

- Touchart L., Bartout L., 2011, « La gestion du risque thermique en étang : le cas de la dérivation », Riscuri si catastrofe, Vol-9, p.149-161.

- Weingertner F., Roussel C., 2010, Pourquoi rétablir la continuité écologique des cours d'eau, Sensibilisation aux politiques publiques, ONEMA, consulté le 20 octobre 2012, URL : http:// www.onema.fr/IMG/pdf/continuite_cours-deau.pdf p.14

\section{Sites consultés :}

- onema.fr, www.onema.fr, consulté en 2012-2013 
- rpfrance.eu, www.rpfrance.eu, site de la Représentation permanente de la France auprès de

l'Union Européenne, consulté en mai 2013

- eaufrance.fr, www.eaufrance.fr, consulté en mai 2013

\section{NOTES}

1. Lors du Colloque International «Les étangs : de la recherche scientifique internationale aux pratiques locales du Berry » tenu les 22, 23 et 24 mai 2012 ; lors d'un terrain d'étude en mars 2013 en Estonie pour le programme franco-estonien Parrot ; lors d'un séminaire tenu en mars 2013 à l'université de Tallinn

2. Directeur de la DDT de l'Indre - entrevue du 25 janvier 2012

3. Id.

4. Terme désignant tout à la fois un plan d'eau stagnant ou une eau courante, un fossé naturel ou anthropique, une eau continentale ou déjà maritime, une eau salée ou douce - (Parlement européen, Conseil de l'Union Européenne, 2000)

5. Source : Touchart et Bartout, 2011, p.153

6. Sources : s.n., 2011 b, p.12 ; s.n., 2011 a, p.16.

7. Pour exemple : Berteaud P., 2005 ; S.n., 2011 b

8. Pour exemple : FORGECO, 2010

9. Touchart L., Bartout P., 2011 ; cf. étude III A

10. "Dans les départements [où cela] est possible sans difficultés majeures ", "y procéder dans les meilleurs délais » (MEDDE, 2015, p.6) ou alors établir une « méthode d'identification des cours d'eau dans les autres territoires » (loc. cit., p.2)

11. Un inventaire scientifique dénombre pas moins de 554000 plans d'eau de moins de 1 are (Bartout et Touchart, 2013)

12. Notice constructeur.

13. Source : calculs d'après données inédites, Millot, $C$.

14. Le coût de l'effacement est estimé à une fourchette très large de 10000 à 100000 euros HT par mètre de chute (Agence de l'Eau Seine-Normandie, 2006).

\section{ABSTRACTS}

In France, apprehension of watercourse is qualitative and environmental and his regulation is powerful. Management ponds on watercourse is highly dependent of the same regulation. More and more promoted, diversion is supposed to reduce ponds damages, to achieve good environmental status. But the lack of definition of watercourse and widespread ignorance of the impact of the diversion, even for different types of ponds can lead to an inadequate management of ponds and / or jeopardize their sustainability. Therefore, it is necessary to clarify the concept of watercourse with a reflection on the regulation and management induced by it as well as the usefulness of the bypass scientific contributions.

En France, la conception des cours d'eau est qualitative et environnementale et leur réglementation très forte. La gestion des étangs sur cours d'eau est fortement dépendante de 
cette même réglementation. Pour favoriser l'atteinte du bon état écologique des cours d'eau, l'aménagement de plus en plus promu est le système de dérivation dont le but est de réduire les méfaits réels et/ou supposés de ces plans d'eau. Mais l'absence de définition des cours d'eau et la grande méconnaissance de l'impact de la dérivation, plus encore selon les différents types d'étangs, peuvent engendrer une gestion inadaptée des étangs et/ou mettre en jeu leur pérennité. Il est donc nécessaire d'éclairer la notion de cours d'eau par une réflexion sur la réglementation et la gestion induite par celle-ci ainsi que d'éclairer l'utilité de la dérivation au moyen d'apports scientifiques.

In Frankreich ist die Wasserwirtschaft qualitativ und ökologisch ausgerichtet und einer starken Regulierung unterworfen. Die Bewirtschaftung von Teichen, die sich auf Wasserstraßen befinden, basiert auf letzteren Vorschriften. Zur Erreichung eines guten ökologischen Zustandes der Gewässer wird immer mehr das Bypass-System gefördert mit dem Ziel, die möglichen und / oder reellen negativen Folgen dieser Wasserflächen zu reduzieren. Die fehlende Definition der Fließgewässer und die große Unkenntnis über die Auswirkungen der Umleitung, inklusivehinsichtlich verschiedenerTeicharten, können ein schlechtes Management der Teiche verursachen und ihre Nachhaltigkeit gefährden. Hieraus ergibt sich die Notwendigkeit, das Konzept der Fließgewässer anhand einer Analyse der Regulierung und der daraus resultierenden Bewirtschaftung, näher zu beleuchten und die Nützlichkeit des Bypass-Systems wissenschaftlich zu überprüfen.

\section{INDEX}

Mots-clés: cours d'eau, dérivation, étangs, gestion, réglementation

Schlüsselwörter: Bewirtschaftung, Regulierung, Teichen, Umleitung, Wasserstraßen

Keywords: diversion, management, ponds, regulation, watercourse

\section{AUTHOR}

\section{CAMILLE MILLOT}

Docteure en Géographie - camille.millot[chez]gmail.com 